\title{
Ladder Operators for the Spherical 3D Harmonic Oscillator
}

\author{
João Marcos Costa Monteiro*1] \\ ${ }^{1}$ Universidade Estadual Paulista, Instituto de Biociências, Letras e Ciências Exatas, \\ São José do Rio Preto, SP, Brasil.
}

Received on September 16, 2020. Revised on November 09, 2020. Accepted on November 18, 2020.

\begin{abstract}
The Schrödinger equation for an isotropic three-dimensional harmonic oscillator is solved using ladder operators. The starting point is the shape invariance condition, obtained from supersymmetric quantum mechanics. Generalized ladder operators can be constructed for the three spherical spatial coordinates. Special emphasis is given to the adaptation made to each of these coordinates. The approach used is general and is indicated as an alternative method to solve the Schrödinger equation.
\end{abstract}

Keywords: Ladder operators, Harmonic oscillator, Shape invariance, Supersymmetric quantum mechanics.

\section{Introduction}

The harmonic oscillator is one of the most fundamental systems studied in quantum mechanics [1-3]. As a first model, any behavior of oscillatory physical systems near the equilibrium position can be described by the harmonic oscillator [1], such as, for instance, diatomic molecules and solids.

In the present work, the Schrödinger equation for the three-dimensional (3D) harmonic oscillator is solved by using the spherical coordinates. An algebraic approach is used to factorize the differential equations and ladder operators are built for each coordinate [4, 5]. This approach is general and it can be used to solve the Schrödinger equation exactly, fitting into the context of Supersymmetric Quantum Mechanics (SQM) [6]. The development of SQM [7, 8] has contributed to increase algebraic methods 9] applied to quantum mechanical problems and the shape invariance property has an underlying algebraic structure associated with Lie algebras [5, 10-13]. The isotropic spherical harmonic oscillator can be used as an introductory problem for formalism and for the construction of generalized ladder operators.

The approach, by using SQM to solve the Schrödinger equation, has already been applied to study different problems, such as, barrier penetration [14], exactly solvable coupled-channels problems [15] and the study of coherent states for unidimensional potentials [16 18]. The supersymmetric formalism has been used to obtain the solution of the Schrödinger equation for the onedimensional harmonic oscillator [19] and for the harmonic oscillator with a position dependent mass [20], besides also being applied to the Dirac oscillator [21]. Furthermore, the use of generalized ladder operators

\footnotetext{
* Correspondence email address: cmonteiro.jm@gmail.com
}

for shape invariant potentials has also been successfully explored to different potentials such as the Coulomb potential and the harmonic oscillator [4, 22, 23. However, the use of these ladder operators for analyzing the radial and the angular coordinates in the same formalism can be further explored. In our best knowledge, this approach was made just for the Coulomb potential [24].

Bazeia and Das 25] solve the Schrödinger equation for the angular coordinate $\theta$ and identify the ladder operators for the Legendre equation. In addition, Dutt et al. 26] explore the shape invariance for the $\theta$-component of the spherical harmonics. However, they do not explore the relations between the shape invariance parameters and the other quantum numbers of the system to determine the harmonic oscillator eigenvalues.

The Schrödinger equation for the $\varphi$ coordinate has been little explored in the literature using generalized ladder operators. This equation is similar to the particle in a box problem and it has a hidden shape invariance [27]. In the current case, from the boundary conditions, two distinct solutions can be obtained. The linear combination of these solutions results in the general solution for this coordinate [24].

In the next section, we introduce the formalism used to construct the generalized ladder operators. In Section 3 , the radial Schrödinger equation for the threedimensional harmonic oscillator is solved using this approach, while in Section 4 the solution to the Schrödinger equation for the angular coordinate $\theta$ is determined. In Section 5 , the solution to the angular coordinate $\varphi$ is obtained. Finally, Section 6 brings the conclusions.

\section{Methodology}

The one-dimensional Schrödinger equation can be written as:

$$
H \psi_{n}(x)=E \psi_{n}(x),
$$


where the Hamiltonian operator can have the convenient form of:

$$
H=-\frac{d^{2}}{d x^{2}}+V(x)
$$

where $\hbar^{2}=2 m=1$, for simplicity. There is no restriction on the nature of the coordinate $x$, i.e. it can be cartesian or not. In Equation (2), $V(x)$ represents the potential and other functions of the respective coordinates, as, for instance, the centrifugal term for the radial coordinate in a central force potential [1].

In a non-relativistic system described by the Schrödinger equation - Equation (1) - the supersymmetric quantum mechanics can be constructed if the Hamiltonian operator $H$ can be factorized in terms of the bosonic operators [4, 8]:

$$
A^{ \pm}=\mp \frac{d}{d x}+W\left(x ; a_{0}\right) .
$$

These bosonic operators are defined in terms of the superpotential $W\left(x ; a_{0}\right)$, which is a function of the position and a set of space-independent parameters $\left\{a_{0}\right\}$. The first supersymmetric partner Hamiltonian is obtained by:

$$
H^{+}=H-E_{0}=A^{+} A^{-},
$$

where $E_{0}$ is the ground state eigenvalue. The second supersymmetric partner Hamiltonian is obtained by changing the bosonic operators:

$$
H^{-} \equiv A^{-} A^{+} \text {. }
$$

The operators $H^{+}$and $H^{-}$have the same energy spectra, except for the ground state of the Hamiltonian $\mathrm{H}^{+}$, for which there is no corresponding state in the $\mathrm{H}^{-}$ spectrum.

From equation (4), the factorization of the Hamiltonian $H$ leads to the Ricatti equation [28]:

$$
W^{2}\left(x ; a_{0}\right)-\frac{d}{d x} W\left(x ; a_{0}\right)=V(x)-E_{0} \equiv V^{+}\left(x ; a_{0}\right),
$$

in which the superpotential is defined. The corresponding potential $V^{-}(x ; a)$, obtained from equation (5), satisfies:

$$
W^{2}\left(x, a_{0}\right)+\frac{d}{d x} W\left(x ; a_{0}\right) \equiv V^{-}\left(x ; a_{0}\right) .
$$

The shape invariance condition [6] imposes that:

$$
R\left(a_{1}\right)=V^{-}\left(x ; a_{0}\right)-V^{+}\left(x ; a_{1}\right),
$$

where the remainder $R\left(a_{1}\right)$ is independent of the coordinates, $V^{+}$is defined with respect to parameter $a_{1}$ and $V^{-}$with respect to parameter $a_{0}$. These parameters are usually related to each other by a translation:

$$
a_{1}=a_{0}+\eta,
$$

in which $\eta$ corresponds to the translation step.

Since the transformation of the parameters involves a translation, we can define the translation operators $T^{ \pm}\left(a_{0}\right)$, as usual, as:

$$
T^{+}\left(a_{0}\right)=\exp \left(\eta \frac{\partial}{\partial a_{0}}\right)
$$

and

$$
T^{-}\left(a_{0}\right)=\exp \left(-\eta \frac{\partial}{\partial a_{0}}\right) .
$$

From the bosonic operators $A^{ \pm}$, equation (3), and the translation operators $T^{ \pm}$, the generalized creation and annihilation operators can be composed as [4, 5]:

$$
B^{+}\left(x ; a_{0}\right) \equiv A^{+}\left(x ; a_{0}\right) T^{+}\left(a_{0}\right)
$$

and

$$
B^{-}\left(x ; a_{0}\right)=T^{-}\left(a_{0}\right) A^{-}\left(x ; a_{0}\right) .
$$

The operators $B^{ \pm}$have the usual algebraic structure of ladder operators, as indicated in reference [5]. In this sense, the factorization method can be used to solve the Schrödinger equation with the shape-invariant potentials [4, 29. Thus, the ground state eigenfunction $\psi_{0}\left(x ; a_{0}\right)$ can be obtained from the application of the generalized annihilation operator $B^{-}$in $\psi_{0}\left(x ; a_{0}\right)$ :

$$
B^{-}\left(x ; a_{0}\right) \psi_{0}\left(x ; a_{0}\right)=T^{-}\left(a_{0}\right) A^{-}\left(x ; a_{0}\right) \psi_{0}\left(x ; a_{0}\right)=0 .
$$

$A^{-}\left(x ; a_{0}\right) \psi_{0}\left(x ; a_{0}\right)=0$ is a sufficient condition to validate the equality in the equation (14). Then, the ground state eigenfunction is written as:

$$
\psi_{0}\left(x ; a_{0}\right) \propto \exp \left(-\int W\left(x ; a_{0}\right) d x\right)
$$

This relation between the ground state eigenfunction and the superpotential is commonly found in the supersymmetric approach [8, 30]. Furthermore, the excited states are obtained by the application of the generalized creation operator in the ground state eigenfunction:

$$
\psi_{n}\left(x ; a_{0}\right)=\left[B^{+}\left(x ; a_{0}\right)\right]^{n} \psi_{0}\left(x ; a_{0}\right) .
$$

To determine the eigenvalues, we start from the shape invariance condition, equation (8), and the operators given by equations 10 and 11 :

$$
R\left(a_{n}\right)=T^{+}\left(a_{0}\right) R\left(a_{n-1}\right) T^{-}\left(a_{0}\right),
$$

in which

$$
a_{n}=a_{0}+n \eta
$$

is a generalization of the relation between the parameters $a_{1}$ and $a_{0}$ given by equation (9). In the same way, it can be written as:

$$
R\left(a_{n}\right) B^{+}\left(x ; a_{n}\right)=B^{+}\left(x ; a_{n}\right) R\left(a_{n-1}\right) .
$$


The commutation relation between operators $H^{+}$and $\left(B^{+}\right)^{n}$ can be determined from the definitions, equations 12 and 13 and the shape invariance condition, equation (8) 44 6]. Then, this gives:

$$
\begin{aligned}
{\left[H^{+},\left(B^{+}\left(x ; a_{0}\right)\right)^{n}\right] } & \\
= & R\left(a_{1}\right)\left(B^{+}\left(x, a_{0}\right)\right)^{n}+R\left(a_{2}\right)\left(B^{+}\left(x, a_{0}\right)\right)^{n} \\
& +\left(B^{+}\left(x ; a_{0}\right)\right)^{2}\left[H^{+},\left(B^{+}\left(x ; a_{0}\right)\right)^{n-2}\right] .
\end{aligned}
$$

Repeating the procedure $n$ times, until $\left[H^{+},\left(B^{+}\right.\right.$ $\left.\left(x, a_{0}\right)^{n-n}\right]=0$, this commutation relation becomes:

$$
\left[H^{+},\left(B^{+}\left(x ; a_{0}\right)\right)^{n}\right]=\left(\sum_{k=1}^{n} R\left(a_{k}\right)\right)\left(B^{+}\left(x ; a_{0}\right)\right)^{n} .
$$

Introducing the commutator in the eigenfunction $\psi_{0}\left(x ; a_{0}\right)$ and noting that for this state $H^{+} \psi_{0}\left(x ; a_{0}\right)=0$, gives:

$$
\begin{aligned}
H^{+}\left[B^{+}\left(x ; a_{0}\right)\right]^{n} \psi_{0}\left(x ; a_{0}\right) \\
\quad=\left(\sum_{k=1}^{n} R\left(a_{k}\right)\right)\left[B^{+}\left(x ; a_{0}\right)\right]^{n} \psi_{0}\left(x ; a_{0}\right) .
\end{aligned}
$$

Notice that $\psi_{0}\left(x ; a_{0}\right)$ is the ground state of the Hamiltonian $H^{+}$with a null eigenvalue. Thus, $\left[B^{+}\left(x ; a_{0}\right)\right]^{n} \psi_{0}\left(x ; a_{0}\right)$ is an eigenfunction of $H^{+}$with eigenvalue $\left(\sum_{k=1}^{n} R\left(a_{k}\right)\right)$. Therefore, the energy eigenvalues from the original Hamiltonian, equation (2), are obtained by:

$$
E_{n}=E_{0}+\left(\sum_{k=1}^{n} R\left(a_{k}\right)\right)
$$

where the $E_{0}$ in this equation is added from the first factorization, equation (4).

In the following sections, the 3D harmonic oscillator is analyzed and the differential equations for each spherical coordinate are solved using the approach described in this section.

\section{The Radial Coordinate}

The Schrödinger equation for the any spherically symmetric potential can be separated into three differential equations, one equation for each spherical coordinate: $r, \theta, \varphi$.

The Schrödinger equation for the radial coordinate is [1]:

$$
\frac{d}{d r}\left(r^{2} \frac{d}{d r} R(r)\right)-\frac{2 m}{\hbar^{2}}[V(r)-E] R(r)=l(l+1) R(r) .
$$

From the substitution of the $R(r)$ function by $\psi(r)=$ $r R(r)$, equation 24 is written as:

$$
-\frac{\hbar^{2}}{2 m} \frac{d^{2} \psi(r)}{d r^{2}}+\left[V(r)+\frac{\hbar^{2}}{2 m} \frac{l(l+1)}{r^{2}}\right] \psi(r)=E \psi(r) .
$$

Then, the radial Hamiltonian for harmonic oscillator can be identified as:

$$
H=-\frac{d^{2}}{d r^{2}}+r^{2}+\frac{l(l+1)}{r^{2}}
$$

in which, for simplicity, it is adopted that $\hbar^{2}=2 m=1$.

The Hamiltonian (26) can be factorized, for each value of $l$, by using the superpotential [4]:

$$
W(r, l)=r-\frac{(l+1)}{r} .
$$

It is worth noting that the quantum number $l$ is used as the parameter $a$, so that $a_{0}=l$. The first supersymmetric Hamiltonian is obtained from equation (4):

$$
H^{+}=-\frac{d^{2}}{d r^{2}}+r^{2}+\frac{l(l+1)}{r^{2}}-2 l_{0}-3=H-E_{0}^{(l)} .
$$

From this equation, we obtain:

$$
E_{0}^{(l)}=2 l+3 .
$$

The supersymmetric partner of the Hamiltonian in equation 28 is:

$$
H^{-}=-\frac{d^{2}}{d r^{2}}+r^{2}+\frac{(l+1)(l+2)}{r^{2}}-2 l-1 .
$$

The shape invariance condition, equation (8), provides:

$$
\begin{aligned}
R\left(l_{1}\right)= & r^{2}+\frac{\left(l_{0}+1\right)\left(l_{0}+2\right)}{r^{2}}-2 l_{0}-1 \\
& -r^{2}-\frac{l_{1}\left(l_{1}+1\right)}{r^{2}}+2 l_{1}+3 .
\end{aligned}
$$

To obtain $R\left(l_{1}\right)$ independent of the spatial coordinate $r$, we must get $l_{1}=l_{0}+1$, implying that $\eta=1$ and so:

$$
R\left(l_{1}\right)=4 \text {. }
$$

It would also be possible to take $l_{1}=-l_{0}-2$ to fulfill the shape invariance condition in equation (31). However, this condition leads to a non-normalizable eigenfunction and it is discarded.

For the $k$ th parameter, we have $l_{k}=l_{0}+k$ from equation (18) and the remainder can be written as:

$$
R\left(l_{k}\right)=-2\left(l_{k}-k\right)-1+2\left(l_{k}-k\right)+2+3=4 .
$$

Then, the energy spectrum can be obtained from equation (23), by using equations 29 and 33 :

$$
E_{n}^{(l)}=E_{0}^{(l)}+\sum_{k=1}^{n} R\left(l_{k}\right)=2 l+3+4 n .
$$

In this case, the generalized ladder operators for the radial coordinate are:

$$
B^{+}(r ; l)=\left[-\frac{d}{d r}+r-\frac{(l+1)}{r}\right] \exp \left(\frac{\partial}{\partial l}\right)
$$


Table 1: Radial eigenfunctions and energy eigenvalues for the spherical harmonic oscillator.

\begin{tabular}{lcc}
\hline$n$ & $\psi_{n}^{(l)}$ & $E_{n}^{(l)}$ \\
\hline 0 & $r^{l+1} e^{-\frac{r^{2}}{2}}$ & $2 l+3$ \\
1 & $\left(2 r^{2}-2 l-3\right) r^{l+1} e^{-\frac{r^{2}}{2}}$ & $2 l+7$ \\
2 & {$\left[4 r^{4}-4(5+2 l) r^{2}+4 l^{2}+16 l+15\right] r^{l+1} e^{-\frac{r^{2}}{2}}$} & $2 l+11$ \\
3 & {$\left[8 r^{6}-12(7+2 l) r^{4}+6(5+2 l)(7+2 l) r^{2}-8 l^{3}-60 l^{2}-142 l-105\right] r^{l+1} e^{-\frac{r^{2}}{2}}$} & $2 l+15$ \\
\hline
\end{tabular}

and

$$
B^{-}(r ; l)=\exp \left(-\frac{\partial}{\partial l}\right)\left[\frac{d}{d r}+r-\frac{(l+1)}{r}\right] .
$$

The ground state eigenfunction for each $l$ is obtained by the substitution of the superpotential, equation (27), into equation (15):

$$
\psi_{0}^{(l)}(r ; l) \propto r^{l+1} e^{-\frac{r^{2}}{2}} .
$$

Excited state eigenfunctions are calculated by the action of the generalized creation operator, equation (35), on the above eigenfunction as indicated in equation (16) 4. Table 1 shows, as an example, the ground state, the first three excited states and their respective eigenvalues for an arbitrary value of $l$. The eigenfunctions are not normalized. In this approach, the eigenfunctions must be normalized at the end. The results are the same as those obtained by other methods, as can be seen in Ref. [3].

\section{The Angular Coordinate $\theta$}

Although not directly related to the energy eigenvalue, the equation expressed in the angular coordinate $\theta$ can also be solved by the approach described in Section 2 The equation for this coordinate is [1]:

$\frac{1}{\sin \theta} \frac{d}{d \theta}\left(\sin \theta \frac{d}{d \theta} \Theta(\theta)\right)+\left(l(l+1)-\frac{m^{2}}{\sin ^{2} \theta}\right) \Theta(\theta)=0$.

In a more convenient form, this equation is equivalent to:

$$
\frac{d^{2}}{d \theta^{2}} \Theta(\theta)+\cot (\theta) \frac{d}{d \theta} \Theta(\theta)+\left[l(l+1)-\frac{m^{2}}{\sin ^{2}(\theta)}\right] \Theta(\theta)=0 .
$$

Equation 39 can be rewritten by the substitution introduced in Ref. [26, changing the variable $\theta$ through a function $\theta=f(z)$, resulting in:

$$
\begin{aligned}
& \left\{\frac{d^{2}}{d z^{2}}+\left[-\frac{f^{\prime \prime}(z)}{f^{\prime}(z)}+f^{\prime}(z) \cot [f(z)]\right] \frac{d}{d z}\right. \\
& \left.+f^{\prime 2}(z)\left[l(l+1)-\frac{m^{2}}{\sin ^{2}[f(z)]}\right]\right\} \Theta(z)=0 .
\end{aligned}
$$

To eliminate the first derivative, it is supposed that:

$$
-\frac{f^{\prime \prime}(z)}{f^{\prime}(z)}+f^{\prime}(z) \cot [f(z)]=0,
$$

which is solved by [26]

$$
\theta \equiv f(z)=2 \operatorname{arctg}\left(e^{z}\right) .
$$

Thus, equation 40 can be written as:

$$
\left[-\frac{d^{2}}{d z^{2}}-l(l+1) \operatorname{sech}^{2}(z)\right] \Theta(z)=-m^{2} \Theta(z) .
$$

The Hamiltonian from equation 43 has the same mathematical structure as the asymmetric Rosen-Morse potential, and it can be factorized by the superpotential 26, 30, 31]:

$$
W\left(z ; a_{0}\right)=a_{0} \tanh (z) .
$$

This way, the first supersymmetric partner is:

$$
H^{+}=-\frac{d^{2}}{d z^{2}}+a_{0}^{2}\left[1-\operatorname{sech}^{2}(z)\right]-a_{0} \operatorname{sech}^{2}(z)=H-\varepsilon_{0}^{(z)} .
$$

From this equation, we can obtain the eigenvalue $\varepsilon_{0}^{(z)}=$ $-a_{0}^{2}=-l^{2}$.

The supersymmetric partner (equation $(\sqrt{5}))$ ) of the Hamiltonian 45 is:

$$
H^{-}=-\frac{d^{2}}{d z^{2}}+a_{0}^{2}\left[1-\operatorname{sech}^{2}(z)\right]+a_{0} \operatorname{sech}^{2}(z) .
$$

The shape invariance condition, equation (8), provides:

$$
R\left(a_{1}\right)=a_{0}^{2}-a_{1}^{2}+\left(a_{1}^{2}-a_{0}^{2}+a_{0}+a_{1}\right) \operatorname{sech}^{2}(z) .
$$

To obtain $R\left(a_{1}\right)$ independent on the $z$ coordinate we must impose that $a_{1}=a_{0}-1$. This condition implies that $\eta=-1$, equation (9), and so:

$$
R\left(a_{1}\right)=2 a_{1}+1 \text {. }
$$

There is another possibility. Choosing $a_{1}=-a_{0}$ fulfills the shape invariance condition in equation (47). However, this condition leads to a non-normalizable eigenfunction. 
For the $k$ th parameter, the shape invariance condition results in:

$$
\begin{aligned}
R\left(a_{k}\right) & =a_{k-1}^{2}-a_{k}^{2}=\left[a_{0}-(k-1)\right]^{2}-\left[a_{0}-k\right]^{2} \\
& =2\left(a_{0}-k\right)+1 .
\end{aligned}
$$

The eigenvalues can be obtained from equation 23 :

$$
\varepsilon_{j}^{(z)}=-l^{2}+\sum_{k=1}^{j}[2(l-k)+1]=-(l-j)^{2} .
$$

Note that these eigenvalues are not the energy of the system.

A comparison between equations 50 and 43 identifies that $\varepsilon_{j}^{(z)}=-m^{2}$, leading to the relation between the parameters $m, j$ and $l$ :

$$
j=l \pm m \text {. }
$$

This relation implies that $m$ is an integer and $-l \leq m<0$ or $0 \leq m \leq l$. Since $j$ is an index of the sum in equation (50), $j>0$.

Summing up, for this coordinate, the generalized ladder operators are:

$$
B^{+}(z ; l)=\left[-\frac{d}{d z}+l \tanh (z)\right] \exp \left[-\frac{\partial}{\partial l}\right]
$$

and

$$
B^{-}(z ; l)=\exp \left[\frac{\partial}{\partial l}\right]\left[\frac{d}{d z}+l \tanh (z)\right] .
$$

In this case, the "ground state" eigenfunction is obtained by substitution of equation (44) into equation 15 :

$$
\Theta_{0}(z ; l) \propto \operatorname{sech}^{l}(z)
$$

It is possible to come back to the original coordinate, $\theta$, by using some algebra. The eigenfunction in the original variable can be written as:

$$
\Theta_{0}(\theta ; l) \propto \sin ^{l}(\theta) .
$$

The generalized creation operator, equation (52), applied in the ground state eigenfunction (54) results in the other eigenfunctions for the original problem [24, 26, 30], equation (38). The eigenvalues analysis implies that $m=0, \pm 1, \pm 2, \pm 3, \ldots, \pm l$, as expected.

\section{The Angular Coordinate $\varphi$}

The Schrödinger equation for the angular coordinate $\varphi$ is [1]:

$$
-\frac{d^{2}}{d \varphi^{2}} \Phi(\varphi)=m^{2} \Phi(\varphi)
$$

This equation is similar to the Schrödinger equation for a particle in an infinite square well. However, boundary conditions are different in these two cases. For the 3D harmonic oscillator, the variable $\varphi$ must be periodical, i.e., $\Phi(\varphi)=\Phi(\varphi+2 \pi)$, while, for the unidimensional infinite square well, the eigenfunction must be null at the walls [1 3]. In addition, we note that the quantum number $m$ is the same as in the previous section, provided by the separation of variables process.

A hidden shape invariance was identified in this kind of problem [27], i.e., a special choice of the parameters $a$ is necessary to identify this invariance and to solve equation $(56)$. The procedure requires parameter $a_{0}$ to be explicitly written in the superpotential. Furthermore, there are two superpotentials that satisfy the Ricatti equation (6) with a null potential (obtained from equation $(\sqrt{56}))$ ) and each one leads to a different solution for the differential equation. The linear combination of these solutions allows us to obtain the exponential form, usually presented for the $\varphi$ variable [1-3].

The first possible solution for the Ricatti equation (6) with $V(\varphi)=0$ is obtained with the following superpotential:

$$
W\left(\varphi ; a_{0}\right)=a_{0} \tan (\varphi)
$$

leading to the supersymmetric partner Hamiltonians:

$$
H^{+}=-\frac{d^{2}}{d \varphi^{2}}+a_{0}\left(a_{0}-1\right) \sec ^{2}(\varphi)-a_{0}^{2}
$$

and

$$
H^{-}=-\frac{d^{2}}{d \varphi^{2}}+a_{0}\left(a_{0}+1\right) \sec ^{2}(\varphi)-a_{0}^{2}
$$

The shape invariance condition provides:

$$
R\left(a_{1}\right)=\left[a_{0}\left(a_{0}+1\right)-a_{1}\left(a_{1}-1\right)\right] \sec ^{2}(\varphi)+a_{1}^{2}-a_{0}^{2} .
$$

By defining the superpotential, equation (57), with $a_{0}=1$ or $a_{0}=0$, these values of $a_{0}$ allow for the factorization of the Hamiltonian in equation 56 , leading to the interpretation that the system is not shape-invariant, which characterizes it as a hidden shape invariance [26]. This invariance is evident if the dependence of the parameter $a_{0}$ is explicitly stated in the superpotential.

To have $R\left(a_{1}\right)$ independent of the spatial coordinate $\varphi$, we must have $a_{1}=a_{0}+1$, implying $\eta=1$ and so:

$$
R\left(a_{1}\right)=2 a_{0}+1
$$

where the $k$ th parameter is given by:

$$
R\left(a_{k}\right)=2 k+1 \text {. }
$$

From equations 58 and 62 and now allowing $a_{0}=1$, we obtain:

$$
m_{n}^{2}=1+\sum_{k=1}^{n}(2 k+1)=(n+1)^{2} .
$$


The index $n$ can be suppressed from equation (63), i.e., $m= \pm 1, \pm 2, \pm 3, \ldots$, and the sign \pm naturally appears by applying the square root to equation 63 . It would also be possible to take $a_{0}=0$. This allows the factorization of the Hamiltonian present in equation (56), leading to null eigenvalues for all states, i.e., $m=0$.

The first eigenfunction can be obtained directly from equation (15) with the superpotential (57):

$$
\Phi_{0}\left(\varphi ; a_{0}\right) \propto \cos ^{a_{0}}(\varphi)
$$

and the generalized ladder operators are written as:

$$
\begin{aligned}
& B^{+}\left(\varphi ; a_{0}\right)=\left[-\frac{d}{d \varphi}+a_{0} \tan (\varphi)\right] \exp \left[\eta \frac{\partial}{\partial a_{0}}\right] \\
& B^{-}\left(\varphi ; a_{0}\right)=\exp \left[-\eta \frac{\partial}{\partial a_{0}}\right]\left[\frac{d}{d \varphi}+a_{0} \tan (\varphi)\right] .
\end{aligned}
$$

The action of the generalized creation operator leads to:

$$
\Phi_{1}\left(\varphi ; a_{0}\right) \propto \cos ^{a_{0}}(2 \varphi)
$$

and after $|m|$ applications, we get:

$$
\Phi_{m}\left(\varphi ; a_{0}\right) \propto \cos ^{a_{0}}(m \varphi),
$$

with $m=0,1,2,3 \ldots$ and $a_{0}=1$.

The second possible solution of the Ricatti equation (6) for this problem is obtained by a superpotential written as:

$$
W\left(\varphi ; a_{0}\right)=-a_{0} \cot (\varphi)
$$

leading to the supersymmetric partner Hamiltonians:

$$
H^{+}=-\frac{d^{2}}{d \varphi^{2}}+a_{0}\left(a_{0}-1\right) \csc (\varphi)-a_{0}^{2},
$$

and

$$
H^{-}=-\frac{d^{2}}{d \varphi^{2}}+a_{0}\left(a_{0}+1\right) \csc (\varphi)-a_{0}^{2} .
$$

The shape invariance condition is given by:

$$
R\left(a_{1}\right)=\left[a_{0}\left(a_{0}+1\right)-a_{1}\left(a_{1}-1\right)\right] \csc (\varphi)+a_{1}^{2}-a_{0}^{2},
$$

which is satisfied by the same condition as before $a_{1}=$ $a_{0}+1$, leading to the same eigenvalues, equation $(63)$.

With the superpotential given by equation (69) we obtain the eigenfunction:

$$
\Phi_{0}\left(\varphi ; a_{0}\right) \propto \sin ^{a_{0}}(\varphi)
$$

and the generalized creation and annihilation operators:

$$
\begin{aligned}
& B^{+}\left(\varphi ; a_{0}\right)=\left[-\frac{d}{d \varphi}-a_{0} \cot (\varphi)\right] \exp \left[\eta \frac{\partial}{\partial a_{0}}\right], \\
& B^{-}\left(\varphi ; a_{0}\right)=\exp \left[-\eta \frac{\partial}{\partial a_{0}}\right]\left[\frac{d}{d \varphi}-a_{0} \cot (\varphi)\right] .
\end{aligned}
$$

The action of $|m|$ times the generalized creation operator results in:

$$
\Phi_{m}\left(\varphi ; a_{0}\right) \propto \sin ^{a_{0}}(m \varphi),
$$

where $m=0,1,2,3 \ldots$ and $a_{0}=1$.

The generalized solution is a linear combination of equations 68) and (76):

$$
\Phi_{m}\left(\varphi ; a_{0}\right)=C_{1} \cos ^{a_{0}}(m \varphi)+C_{2} \sin ^{a_{0}}(m \varphi) \propto e^{i m \varphi},
$$

where $a_{0}=1, C_{1}$ and $C_{2}$ are constants and $m=$ $0,1,2,3 \ldots$ The exponential form in equation $\sqrt{77}$ is the usual shape for the $\varphi$-component dependence on spherical harmonics [1-3].

\section{Conclusion}

In this paper, an algebraic method to solve the Schrödinger equation, based on a generalization of ladder operators, is presented. The methodology presented determines the eigenfunctions and the energy eigenvalues of a bond-state Schrödinger equation for any shapeinvariant potential. The approach adopted consists in the construction of generalized ladder operators due the shape invariance condition obtained from SQM formalism.

Solutions for the three spherical coordinates of the three-dimensional harmonic oscillator are obtained. For the $\varphi$-component, a hidden shape invariance is identified. So, the solution for $\varphi$ requires more attention to provide the correct result, as shown in Section 5

Usually, only the radial part of the Schrödinger equation is obtained through the methodology presented here. Recently, the authors 24] proposed a similar approach to obtain the hydrogen eigenvalues and eigenfunctions. As expected, the solutions in that case and in the present analysis are the same obtained from the standard calculation, for example, from Frobenius method.

In short, the energy eigenvalues for the harmonic oscillator are obtained from equation (34) and the restrictions on the quantum numbers $l$ and $m$ are identified. The eigenfunctions $\Psi_{n, l, m}(r, \theta, \varphi)=\psi_{n, l}(r) \Theta_{l, m}(\theta) \Phi_{m}(\varphi)$ are determined from the generalized creation operator applied in the base eigenfunctions. Notice that for the angular coordinate $\theta$ it is necessary to use the relation between the $z$ and $\theta$, to get the usual eigenfunctions. For the $\varphi$ coordinate, the eigenfunctions are obtained by a linear combination of the trigonometric functions.

Finally, we observe that the formalism presented here to obtain the exact solution from the three-dimensional isotropic harmonic oscillator can be used to study other quantum mechanical problems.

\section{Acknowledgment}

This study was financed in part by the Coordenação de Aperfeiçoamento do Pessoal de Nível Superior - Brasil (CAPES) - Finance Code 001. 


\section{References}

[1] D.J. Griffiths, Introduction to Quantum Mechanics (Pearson Prentice Hall, New Jersey, 2004), 2nd ed.

[2] L.I. Schiff, Quantum Mechanics (McGraw-Hill Book Company, New York, 1949), 3rd ed.

[3] A.S. Davydov, Quantum Mechanics: International Series in Natural Philosophy (Pergamon, Massachusetts, 1986), 2nd ed.

[4] E. Drigo Filho and M. A. Cândido Ribeiro, Physica Scripta 64, 548 (2001).

[5] Y. Chen and M.E.H. Ismail, J. Phys. A: Math. Gen. 30, 7817 (1997).

[6] L. Gendenshtein, Pis'ma Zh. Eksp. Teor. Fiz. 38, 299 (1983).

[7] R. W. Haymaker and A.R.P. Rau, Am. J. Phys. 54, 928 (1986).

[8] F. Cooper, A. Khare and U.P. Sukhatme, Phys. Rep. 251, 267 (1995).

[9] E. Schrödinger, Proc. Roy. Irish Acad. 46, 9 (1940).

[10] T. Fukui and N. Aizawa, Phys. Lett. A. 57, 308 (1993).

[11] A. Gangopadhyaya, J.V. Mallow and U.P. Sukhatme, Proc. Workshop on Supersymmetric Quantum Mechanics and Integrable Models (Springer, Berlin, 1997).

[12] S. Chaturvedi, R. Dutt, A. Gangopadhyaya, P. Panigrahi, C. Rasinariu and U.P. Sukhatme, Phys. Lett. A 248, 109 (1998).

[13] A.B. Balantekin, M.A. Cândido Ribeiro and A.N.F. Aleixo, J. Phys. A: Math. Gen. 32, 2785 (1999).

[14] A.N.F. Aleixo, A.B. Balantekin and M.A. Cândido Ribeiro, J. Phys. A: Math. Gen. 33, 1503 (2000).

[15] A.N.F. Aleixo, A.B. Balantekin and M.A. Cândido Ribeiro, J. Phys. A: Math. Gen. 33, 3173 (2000).

[16] D.J. Fernandes, V. Hussin and L.M. Nieto, J. Phys. A: Math. Gen. 27, 3547 (1994).

[17] M.S. Kumar and A. Khare, Phys. Lett. A 217, 73 (1996).

[18] A. Perelomov, Generalized coherent states and their applications (Springer, Berlin, 1986).

[19] C.V. Sukumar, J. Phys. A: Math. Gen. 18, 2917 (1985).

[20] R. Koç and R. Tütüncüler, Ann. Phys. 12, 685 (2003).

[21] C. Quesne, Int. J. Mod. Phys. A 6, 1567 (1991).

[22] N.A. Alves and E. Drigo Filho, J. Phys. A: Math. Gen. 21, 3215 (1988).

[23] Y. Ding, J. Phys. A: Math. Gen. 20, 6293 (1987).

[24] J.M.C. Monteiro, A. Algozini Junior and E. Drigo Filho, Rev. Bras. Ens. Fís. 41, e20180315 (2019).

[25] D. Bazeia and A. Das, Phys. Lett. B 715, 256 (2012).

[26] R. Dutt, A. Gangopadhyaya and U.P. Sukhatme, Am. J. Phys. 65, 400 (1997).

[27] E. Drigo Filho and R.M. Ricotta, J. Phys. A: Math. Gen. 37, 10057 (2004).

[28] S. Bittanti, A.J. Laub and J.C. Willems, The Ricatti Equation (Springer - Verlag, New York, 2012).

[29] G. Junker, Supersymmetric Methods in Quantum and Statistical Physics (Springer International Publishing, Switzerland, 1996).

[30] R. Dutt, A Khare and U.P. Sukhatme, Am. J. Phys. 56, 163 (1988).

[31] H. O. Batael, J.F. Silva, A.N. Silva, S.F.M. Santos and E. Drigo Filho, Rev. Bras. Ens. Fís. 40, e2305 (2018). 